\title{
Exercise and the Eye: A Call for Prospective, Outcomes-Based Research Collaborations between Exercise Physiologists and Ophthalmologists
}

\section{David M Kleinman*}

Flaum Eye Institute, University of Rochester Medical Center, USA

*Corresponding author: David M. Kleinman, MD, MBA, FACS, Associate Professor of Ophthalmology, Flaum Eye Institute, University of Rochester Medical Center, 601 Elmwood Avenue, Box 659, Rochester, New York 14642, USA, Tel: 585-2750626, Fax: 585-276-0292, E-mail: David_Kleinman@URMC.rochester.edu

The benefits of physical activity on health are becoming increasingly understood. Evidence suggests regular exercise leads to better overall heath, lower blood pressure, less chronic pain, reduced risks of cardiovascular events, and improved cognitive functioning [1,2]. These factors overlap with ophthalmic health, and loss of vision is one of the most feared health consequences patients describe. As ophthalmologists well know, recommendations that relate to eyesight carry particular weight, and a better understanding of the relationship between exercise and long-term eye health could become an important facet of a comprehensive approach to motivate patients to improve their health through exercise. Such a development should have important implications for our society and population health.

Ophthalmologists increasingly see aging patients with multiple medical problems. Patients in these cohorts show progressive diabetic retinopathy, age-related macular degeneration (AMD), glaucoma and retinal vascular occlusions - conditions which combined represent the vast majority of blindness and visual disability in developed countries [3]. Interestingly, all these conditions can be tied to the relationship between exercise and its benefits on systemic health [4-7].

Despite this relationship, there is very little prospective clinical information on the effects of exercise on objective, long-term visual function. There does exist epidemiologic data suggesting that individuals who are more physically active or exercise regularly have lower rates of progression of diabetes and incidence of $A M D$ [4,8-11]. Furthermore, elevated blood pressure is a risk factor for glaucoma, elevated intraocular pressure, retinal vascular occlusions, and macular degeneration so it is logical to infer that exercise and the secondary benefits of lowering blood pressure through exercise should also lower rates of vision loss from these conditions $[12,13]$. Of course, other mechanisms of benefit from exercise are likely, as well [7].

Short term studies have at looked ocular blood flow after exercise, and increased retinal tissue blood flow following exercise has been documented [14]. Furthermore, currently, high quality methodology is readily available for assessing retinal and choroidal vasculature, retinal perfusion, the size and extent of macular drusen and atrophy, diabetic macular edema, and of course, visual acuity and visual field function. See Figures 1-6 below, which demonstrate different types of objective and meaningful clinical assessments available to ophthalmologists.

Although excellent intravitreal therapies have been developed for late stage diabetic eye disease, wet macular degeneration, and the secondary effects of retinal vascular occlusions, and eye drops that lower intraocular pressure can help preserve vision in patients with glaucoma, these interventions tend to only slow visual decline, require multiple treatments, carry risk, and are expensive. Furthermore, outside of a specific nutraceutical formulation (as determined by results of

Citation: Kleinman DM (2018) Exercise and the Eye: A Call for Prospective, Outcomes-Based Research Collaborations between Exercise Physiologists and Ophthalmologists. Int J Sports Exerc Med 4:094. doi.org/10.23937/2469-5718/1510094

Accepted: July 03, 2018; Published: July 05, 2018

Copyright: (c) 2018 Kleinman DM. This is an open-access article distributed under the terms of the Creative Commons Attribution License, which permits unrestricted use, distribution, and reproduction in any medium, provided the original author and source are credited. 


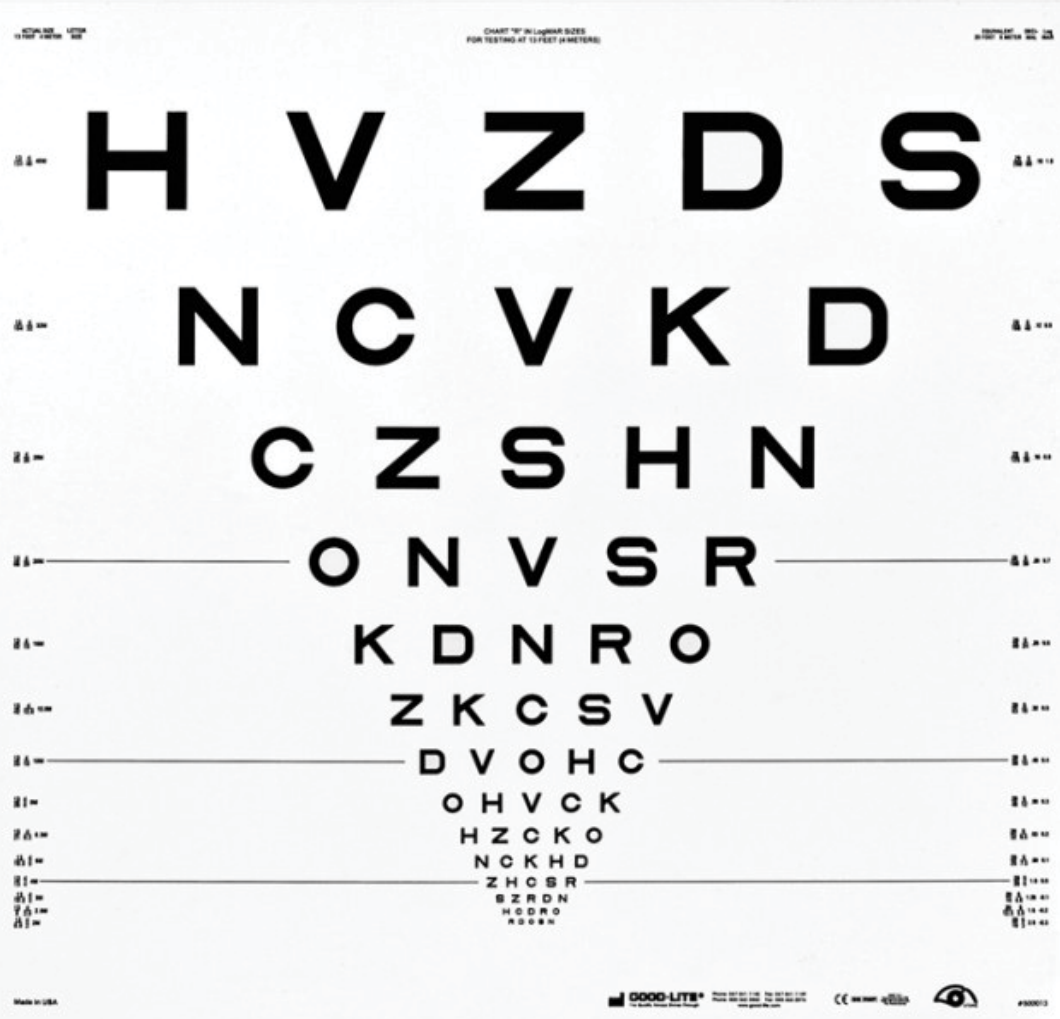

Figure 1: ETDRS visual acuity chart which is the gold standard for obtaining reliable visual acuities. Patients are tested wearing best-correction spectacles at a set distance under standardized illumination. The visual acuity is scored with numbers of letters read correctly which allows for extensive and accurate statistical analyses.

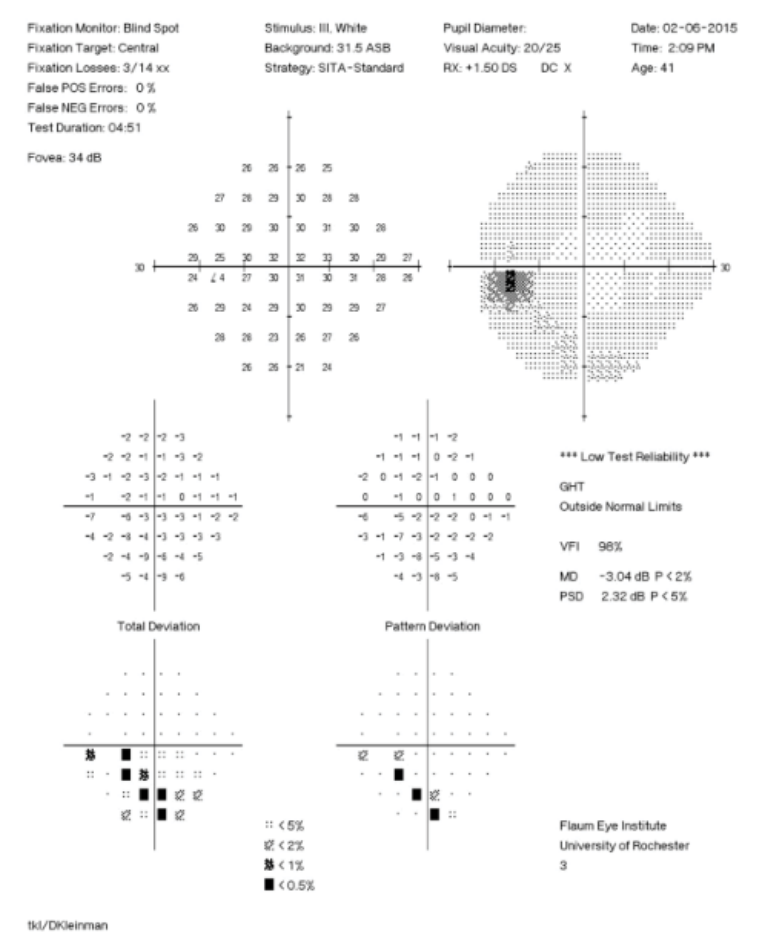

Figure 2: Humphrey Visual Field test results can be used over time to assess for stabilization or progression of the patient's visual field. [Images courtesy of Flaum Eye Institute.]

the Age-Related Eye Disease Studies sponsored by the National Institutes of Health) for a subset of macular degeneration patients, there are no proven therapies currently available to slow the progression of dry macular degeneration.
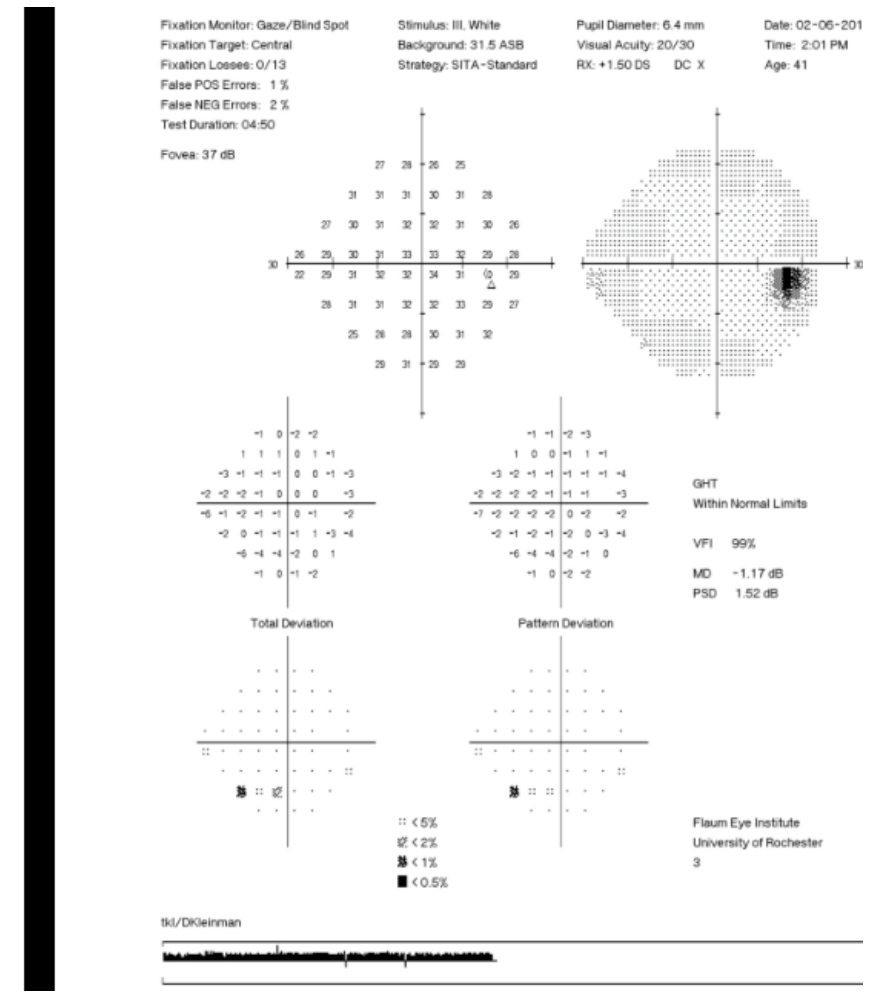


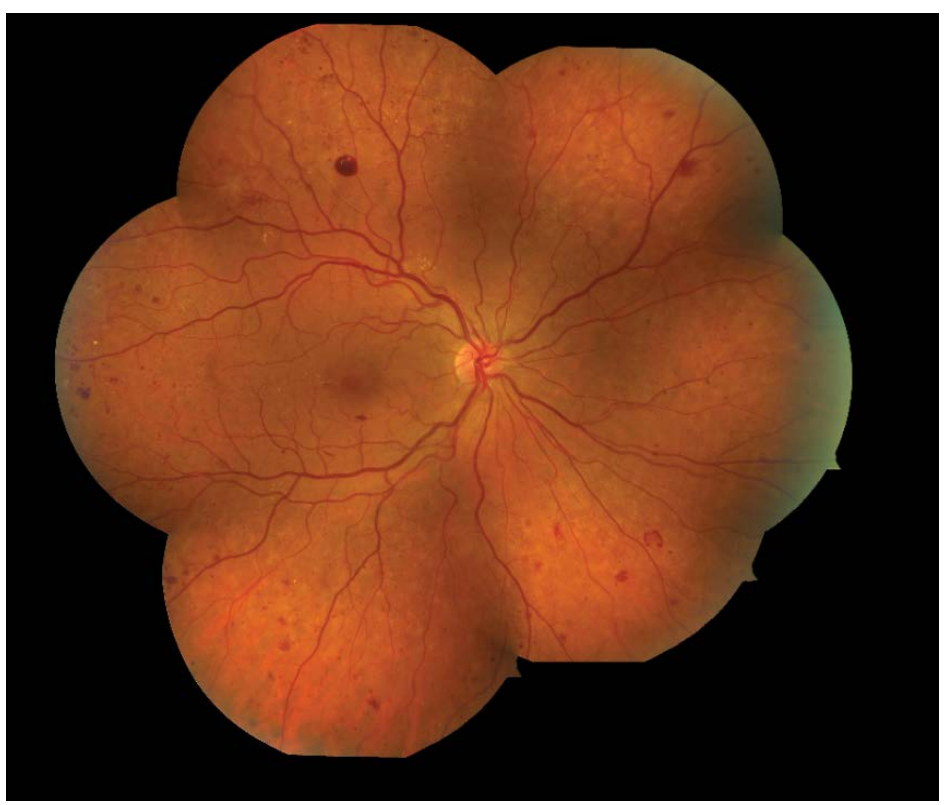

Figure 3: Fundus photography can be used serially to assess for improvement or worsening of diabetic retinopathy. Images are graded against standard photos. Intravenous fluorescein angiography and indocyanine green angiography (not shown) are also photographic techniques for evaluating retinal and choroidal blood flow. [Images courtesy of Flaum Eye Institute.]
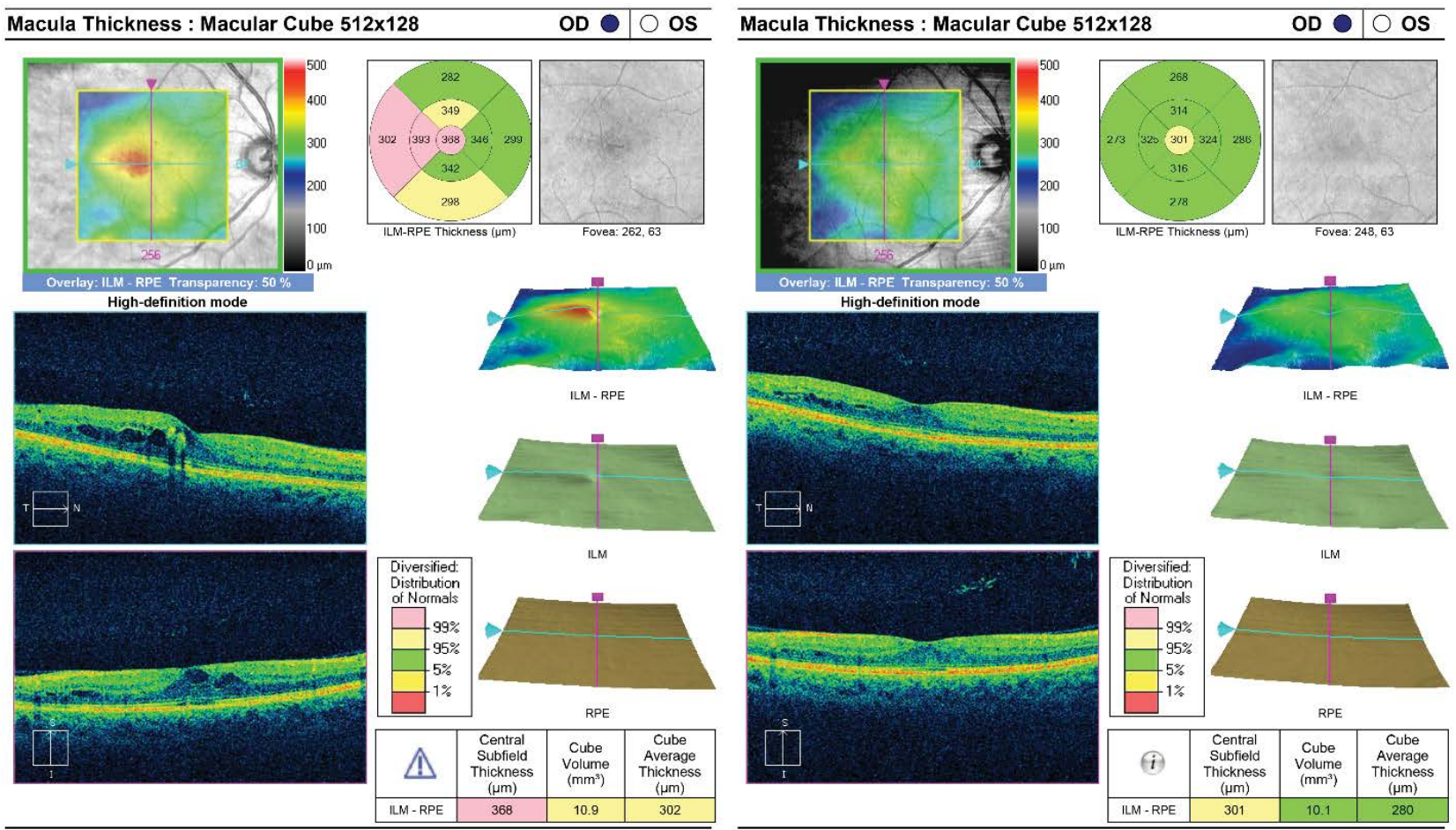

Figure 4: Optical coherence tomography images in diabetic macular edema. Retinal thickness can be accurately quantified, and changes can be documented. [Images courtesy of Flaum Eye Institute.]

types including pediatricians, internists, general practitioners, hospitalists, geriatricians, and non-physician health-care providers. In other words, data correlating exercise with improved visual outcomes can be used by the entire spectrum of health workers as care shifts to population-based health. The adage that prevention is the best cure reigns high in ophthalmology, and as a health care community we need to better inform ourselves and our patients regarding low risk, high reward lifestyle changes that can decrease rates of visual loss.

Toward that end, we need better collaboration be- tween exercise scientists and clinical ophthalmologists. Exercise scientists have vast knowledge about the methods and best approaches to clinical exercise research and the related physiologic responses. Similarly, clinical ophthalmologists have access to the patient populations and the tools necessary to assess the effects of such an intervention on eye health over both short and longterm time frames. A comparison or control group will be very important to increase the validity of study results. These clinical investigations should use a clinically meaningful primary endpoint such as the Early Treatment of 

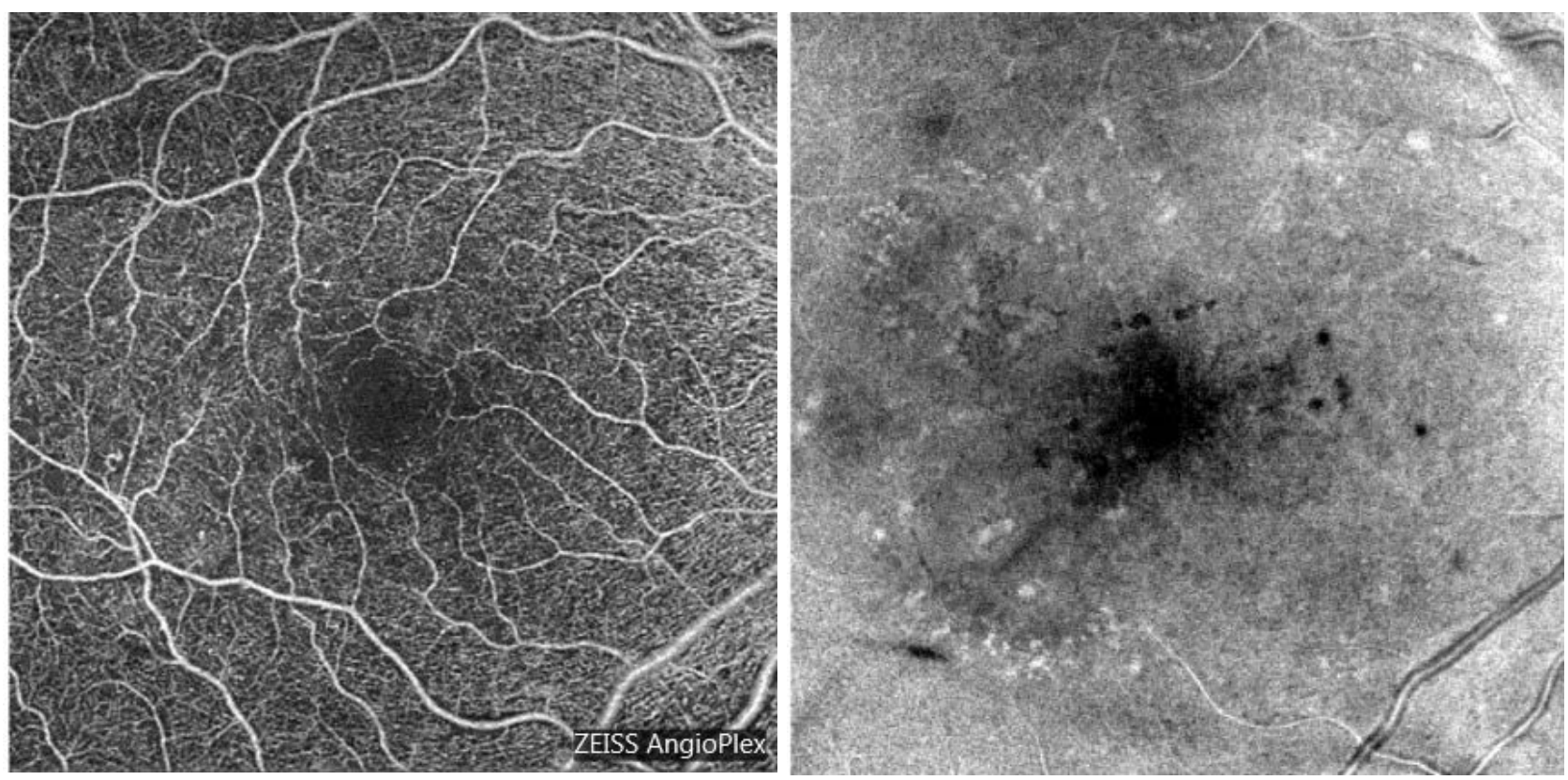

Figure 5: Optical coherence tomography - angiography is a fast, noninvasive method to assess retinal capillaries and choroidal structure. [Images courtesy of Flaum Eye Institute.]

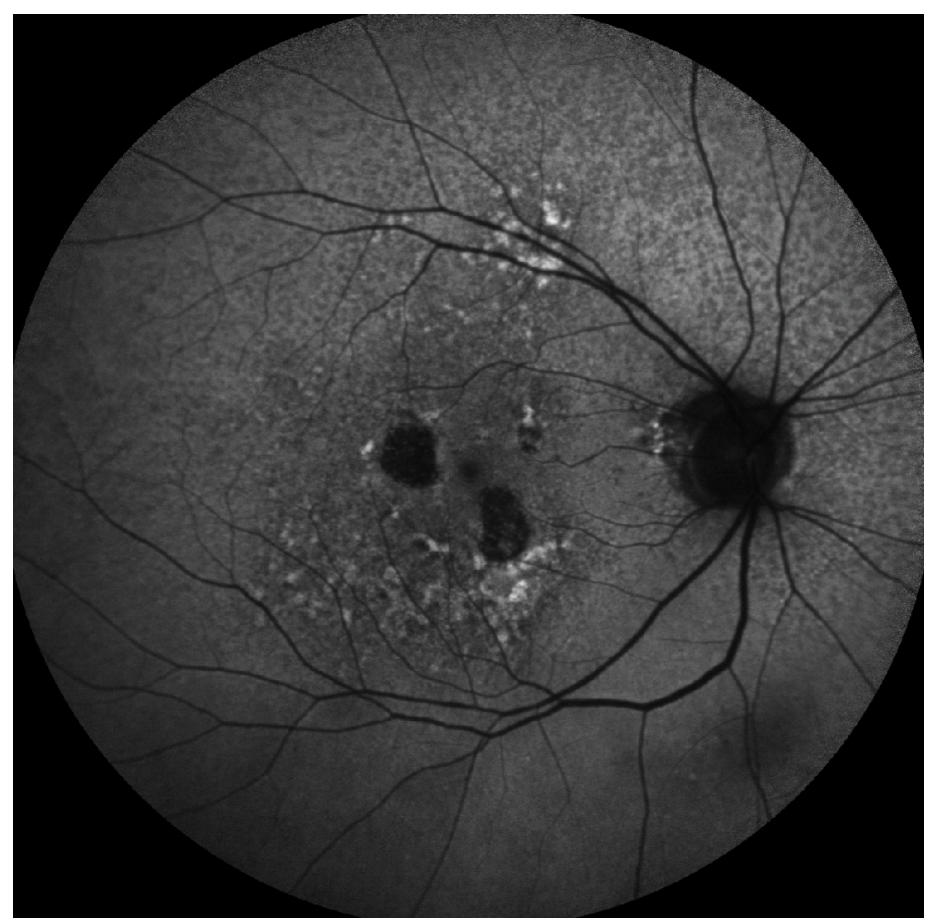

Figure 6: Fundus autofluorescence images can be used to accurately measure extent of drusen and areas of geographic atrophy in AMD. The slowing of rates of progression of area of geographic atrophy is an approvable endpoint for pivotal drug trials. [Images courtesy of Flaum Eye Institute.]

Diabetic Retinopathy Study (ETDRS) best-corrected visual acuity or automated visual field assessments. Secondary outcomes can look at retinal thickness by OCT, retinal perfusion, diabetic retinopathy progression, and size and extent of macular drusen and atrophy.

Once there is good prospective data correlating exercise and visual function, the relationships of exercise to these secondary outcome measures can be utilized in shorter term trials. Such targeted studies can then prospectively assess the effect of a specific physical activity intervention using a surrogate marker, or in other words, a proven predictor for long term visual function. If it can be shown that exercise generally leads to the regression of macular drusen at two years, and the likely cause (evident by ancillary testing at multiple time points) is an increase in choroidal perfusion in the intervention group vs. control group, then the door is now opening to look for similar choroidal perfusion benefits related to a set of activities over a shorter time frame [15]. There could be great interest in evaluating specific behaviors and their influence on these more easily evaluable surrogate markers. For example, is the benefit of 
exercise on the eye related to basic physiologic changes shared by many activities (e.g. increased heart rate, basic measures of exertion) or are there specific exercises that confer more benefit (e.g. swimming, biking, walking, running, or yoga). Interestingly, even mild yoga increases muscle mass, balance, burns calories, and leads to short term increases in heart rate. These benefits may be indistinguishable from the benefits of other forms of mild to moderate physical activity based on data from larger and longer term clinical outcomes studies. Yoga, however, also has the added component of positional inversions where practitioners routinely place their head below their heart. With correlative data of visual outcomes to shorter-term endpoints, more information can be gleaned to help tease out the likely long-term benefits of very specific activities. Of course, the place to start is to increase our understanding of physical activity and functional vision-related endpoints over time.

Here is a list of potential prospective interventional study ideas assessing the relationship between exercise and ophthalmic function that could be valuable to the medical community:

- Does regular exercise correlate with better visual function in aging adults over a set time such as five years?

- Is regular exercise more beneficial in subgroups already predisposed to vision loss (e.g. diabetics or those with $\mathrm{AMD}$ ) in terms of visual acuity testing?

- Can exercise improve or delay the progression of diabetic retinopathy?

- Can exercise enhance the resolution of diabetic macular edema?

- Can exercise lead to a regression of macular drusen in AMD?

- Can exercise slow the growth of geographic atrophy in patients with late stage AMD?

- Can exercise help improve patient outcomes following branch or central retinal vein occlusion?

- Can exercise help delay the loss of the peripheral visual field in patients with glaucoma?

There is rationale to suggest that exercise should stabilize retinal and optic nerve perfusion and enhance retinovascular health. Such a documented outcome in any or all the study scenarios above will provide an added layer of knowledge as physicians advise patients on long term healthy aging and maintaining their sight for life.

Designing, funding, implementing, and executing these trials will be challenging. To be powered appropriately and informative, these studies may require hundreds, if not thousands, of participants. Establishing intervention and control regimens as well as randomization methodologies and stratification variables will require significant thought and planning. Aging cohorts may have difficulties with certain types of exertional activity. Likewise, patients with musculoskeletal comorbidities may be restricted behaviorally. Furthermore, these efforts will likely need to be broadly collaborative and require multiple investigational sites. Not only should participants be evaluated physiologically and systemically regularly, but extensive diagnostic ophthalmic testing will be needed. Investigators should include general medical physicians, exercise physiologists, and ophthalmologists. These studies will not be inexpensive, and external financing whether from governmental sources, charitable foundations, insurance companies, or industry will be required. The societal benefits from this type of intensive investigation will be extremely valuable, so it is likely that when proposed appropriately the financing should be available. Currently, ophthalmologists can partner with pharmaceutical companies to carry out elaborate long-term interventional trials, often with extensive monthly visitation requirements and monthly procedures such as intravitreal injections. It is not hard to imagine that the same quality of study can be carried out with the alternative dependent variable of exercise. These proposed studies will be complex, and behavioral studies certainly have inherent challenges. Although a topic for another venue, evidence suggests dietary choices influence progression of chronic ocular disease as well. Success in the arena of prospectively studying physical activity and eye disease may open doors for similar type designs based on dietary interventions.

Rates of visual impairment and blindness are increasing in the United States [16]. Knowledge gained from prospective ocular health and exercise studies may prove helpful for individual patient as well as population health management, and lower health care costs could be an additional long-term benefit. There are compelling reasons to move forward in this regard.

There is great opportunity for exercise researchers and ophthalmologists to reach out to each other to help move collaborative studies, like those mentioned above, from the white board to the clinic.

\section{References}

1. Bourne RR, Stevens GA, White RA, Smith JL, Flaxman SR, et al. (2013) Causes of vision loss worldwide, 1990-2010: a systematic analysis. Lancet Glob Health 1: 339-349.

2. Haskell WL, Lee IM, Pate RR, Powell KE, Blair SN, et al. (2007) Physical activity and public health: updated recommendation for adults from the American College of Sports Medicine and the American Heart Association. Med Sci Sports Exerc 39: 1423-1434.

3. Warburton DE, Nicol CW, Bredin SS (2006) Health benefits of physical activity: the evidence. Canadian Medical Association Journal 174: 801-809.

4. Knudtson MD, Klein R, Klein BE (2006) Physical activity and the 15-year cumulative incidence of age-related macular degeneration: the Beaver Dam Eye Study. Br J Ophthalmol 90: 1461-1463. 
5. Dirani M, Crowston JG, Wijngaarden P (2014) Physical inactivity as a risk factor for diabetic retinopathy? A review. Clin Exp Ophthalmol 42: 574-581.

6. Pan XR, Li GW, Hu YH, Wang JX, Yang WY, et al. (1997) Effects of diet and exercise in preventing NIDDM in people with impaired glucose tolerance. The Da Qing IGT and Diabetes Study. Diabetes Care 20: 537-544.

7. Gale J, Wells AP, Wilson G (2009) Effects of exercise on ocular physiology and disease. Surv Ophthalmol 54: 349-355.

8. Praidou A, Harris M, Niakas D, Labiris G (2017) Physical activity and its correlation to diabetic retinopathy. J Diabetes Complications 31: 456-461.

9. Kuwata $\mathrm{H}$, Okamura $\mathrm{S}$, Hayashino $\mathrm{Y}$, Tsujii $\mathrm{S}$, Ishii $\mathrm{H}$, et al. (2017) Higher levels of physical activity are independently associated with a lower incidence of diabetic retinopathy in Japanese patients with type 2 diabetes: A prospective cohort study, Diabetes Distress and Care Registry at Tenri (DDCRT15). PLoS One 12: e0172890.

10. McGuinness MB, Le J, Mitchell P, Gopinath B, Cerin E, et al. (2017) Physical activity and age-related macular degeneration: a systematic literature review and meta-analysis. Am J Ophthalmol 180: 29-38.
11. McGuinness MB, Karahalios A, Simpson JA, Guymer $\mathrm{RH}$, Robman LD, et al. (2016) Past physical activity and age-related macular degeneration: the Melbourne Collaborative Cohort Study. Br J Ophthalmol 100: 1353-1358.

12. Kokkinos PF, Narayan P, Papademetriou V (2001) Exercise as hypertension therapy. Cardiol Clin 19: 507-516.

13. Pescatello LS, Franklin BA, Fagard R, Farquhar WB, Kelley GA, et al. (2004) American College of Sports Medicine position stand. Exercise and hypertension. Med Sci Sports Exerc 36: 533-553.

14. Okuno T, Sugiyama T, Kohyama M, Kojima S, Oku H, et al. (2006) Ocular blood flow changes after dynamic exercise in humans. Eye (Lond) 20: 796-800.

15. Sallo FB, Rechtman E, Peto T, Stanescu-Segall D, Vogt $\mathrm{G}$, et al. (2009) Functional aspects of drusen regression in age-related macular degeneration. $\mathrm{Br} \mathrm{J}$ Ophthalmol 93: 1345-1350.

16. Varma R, Vajaranant TS, Burkemper B, Wu S, Torres M, et al. (2016) Visual Impairment and Blindness in Adults in the United States: Demographic and Geographic Variations From 2015 to 2050. JAMA Ophthalmol 134: 802-809. 